\title{
A theoretical and experimental investigation on free vibration behavior of a cantilever beam with a breathing crack
}

\author{
M. Rezaee* and H. Fekrmandi \\ Mechanical Engineering Department, Faculty of Mechanical Engineering, University of Tabriz, Tabriz, Iran
}

Received 20 March 2010

Revised 6 December 2010

\begin{abstract}
In this paper the free nonlinear vibration behavior of a cracked cantilever beam is investigated both theoretically and experimentally. For simplicity, the dynamic behavior of a cracked beam vibrating at its first mode is simulated using a simple single degree of freedom lumped parameter system. The time varying stiffness is modeled using a harmonic function. The governing equation of motion is solved by a perturbation method - the method of Multiple Scales.

Results show that the correction term that is added to the main part of the response reflects the effect of breathing crack on the vibration response. Moreover, this part of response consists of the superharmonic components of the spectrum which is due to the system's intrinsic nonlinearity. Using this method an analytical relation is established between the system characteristics and the crack parameters in one hand and the nonlinear characteristics of system response on the other hand. Results have been validated by the experimental tests and a numerical method.
\end{abstract}

Keywords: Cracked beam, breathing crack, superharmonic components, multiple scales method

\section{Introduction}

In recent years a lot of attention has been made toward developing non-destructive testing methods. Among these techniques, vibration based methods offer an effective and fast means of detecting faults in structures. In order to identify structural damage by vibration monitoring, the study of the changes of the structural dynamic behavior due to cracks is required for developing the detection criteria.

Many researchers in their investigations on cracked beams have used open crack models [1]. Unfortunately, relying on the drop in the natural frequencies only and using the open crack model could lead to underestimating the severity of the crack [2]. Furthermore, as it has been shown that the modal frequencies are not very sensitive to the presence of the breathing cracks, alternative detection techniques have been proposed [3].

The simplest nonlinear model for the stiffness of the cracked beam assumes that the crack is fully closed in one half of the vibration cycle and suddenly shifts to the fully open situation for the other half vibration cycle and back again, which is referred to as the bilinear model. Such a fatigue crack model, however, only represents an idealized situation in which the crack has two perfectly flat surfaces and can only exist in the fully open or fully closed states. In reality, change between these extremes occurs in a smooth way which results in continuous and gradual change of the cracked beam stiffness between the stiffness values corresponding to the fully open and the fully closed crack situations $[4,5]$.

\footnotetext{
*Corresponding author. E-mail: m_rezaee @ tabrizu.ac.ir.
} 
The effect of the breathing crack in the vibration response of cracked structural members had been recognized long ago [6]. Various studies performed over the last two decades have indicated that a beam with a fatigue crack shows non-linear behavior because of the variation in the structural stiffness which is due to the opening and closing the crack [4,5,7-10]. The main distinctive feature of this behavior is the presence of higher harmonic components in the system dynamic response [11]. Moreover, it has been shown that the nonlinear effects are more sensitive to the presence of fatigue cracks in the structure than the change in natural frequencies, or mode shapes [9]. Consequently, it seems appropriate to study the nonlinear dynamic behavior of structures with breathing cracks for developing more accurate vibration based detection techniques.

Several researchers have addressed the problem of a beam with a breathing crack. A simple single degree of freedom (SDOF) model of cracked beam with bilinear stiffness was proposed by Friswell and Penny [12]. Their analysis revealed the appearance of higher harmonics in the response spectrum which are integer multiples of the fundamental frequency. Shen and Chu [13] derived a bilinear equation of motion for each vibration mode of a beam with a breathing crack using the Galerkin method. They analyzed the response spectra in order to detect the changes which are potentially useful for damage assessment. Later the same authors [14], in order to reduce the computational effort, used a closed-form solution based on the use of two square-wave functions to model stiffness changes for bilinear oscillators, and simulate the cracked beam under the low frequency excitation. Ostachowicz and Krawczuck [15] examined the possibility of crack detection on the basis of changes of the higher harmonics in the frequency spectrum. Moreover, the investigations based on the FEM method have confirmed the presence of higher harmonic components and their sensitivity to damage parameters [16-18]. In most studies it is assumed that the crack opens and closes instantaneously while there is experimental evidence that the passage from closed crack to open crack and vice versa happens in a smoother way [19]. Due to this fact, Cheng et al. [5] have proposed a simple harmonic function to model the time varying stiffness of the beam with a breathing crack. Although, the results showed the presence of higher harmonics in the system response, the numerical approach which was used to identify the response did not exhibit the relation between these components and the crack parameters. Based on this model, Douka et al. [20] have investigated the dynamic behavior of a cantilever beam with a breathing crack. They used a time-frequency method as an alternative to Fourier analysis methodology. It is shown that the Instantaneous Frequency fluctuates between the frequencies corresponding to open and closed crack states. In a recent study Peng et al. [21] have introduced new concept of nonlinear output frequency response functions (NOFRFs) to detect cracks in beams using frequency domain information. Results indicated that the NOFRFs are a quite sensitive indicator for the presence of cracks in the beam.

Despite the progress made towards the modeling of a breathing crack, work remains to be done to highlight the complicated opening and closing phenomenon and the consequent variation of physical system parameters and dynamic response. The aim of this paper is to inspect the effect of the nonlinearity on vibration response of a beam with a fatigue crack using a perturbation method as an alternative to common numerical methods. To this end a SDOF model of a cracked cantilever beam is developed. The basic assumption is that the beam vibrates at its fundamental mode. To model the time-varying stiffness of the beam, the simple periodic function proposed by Cheng et al. [5] is adopted. The free vibration response is obtained by the method of Multiple Scales. Results show that the response is composed of a main part and a correction term which reflects the effect of the breathing crack. This term's frequency is two times of the system frequency which means it is the first superharmonic component. It is shown that the variation of the correction term increases with increasing the crack depth and with approaching the crack location to the clamped end which means increasing the effect of the breathing crack on the response. So using the presented method it is possible to establish the analytical relation between the higher harmonic components, system characteristics and the crack parameters. Moreover, the comparison between the obtained response with the one obtained from the numerical technique -the fourth/fifth order Runge-Kutta method- shows excellent agreement for a large variety of system parameters. Finally, some experimental tests were carried out on a beam with a real fatigue crack. The Fast Fourier Transform (FFT) is applied to the measured response. The experimental results are in good agreement with predictions confirming the presence of higher harmonics due to the system nonlinearity. The introduced method allows an efficient and accurate description of the nonlinearities of the system and can be used to improve the reliability and effectiveness of the vibration based crack diagnostic techniques. 

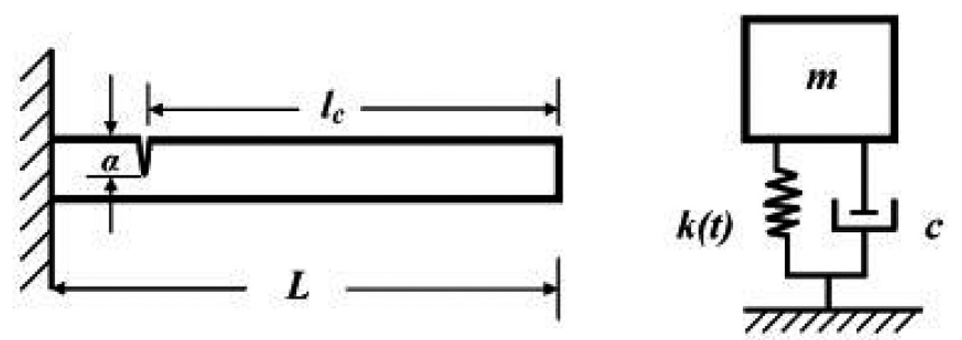

Fig. 1. The cracked cantilever beam and the SDOF lumped parameter model.

\section{SDOF model of a beam with a breathing crack}

In this work, the lateral vibration of a cantilever beam with a breathing crack is modeled as a single degree of freedom lumped parameter system (Fig. 1). This modeling procedure is based on the equalizing the two system's kinetic and potential energies. The fundamental assumption is that the beam vibrates at its fundamental mode. This assumption depends on the kind of initial conditions. In our case, the beam is considered to be displaced at its free end and then is released. In this way, the fundamental mode is predominantly excited. Furthermore, this type of excitation can be easily realized during experimental testing. The modeling procedure that has been employed in this study is capable of modeling the vibration of each point on the beam as the response of a lumped parameter system. In this study, the vibration of the free end of the cracked cantilever beam is considered.

Cheng et al. [5] proposed a simple periodic function which considers the stiffness variation due to the breathing crack, as a simple harmonic function. This model is adopted for the present study and can be expressed in the form:

$$
k(t)=k_{o}+k_{\Delta}\left[1+\cos \left(\omega_{b} t\right)\right]
$$

With:

$$
k_{\Delta}=\frac{k_{c}-k_{o}}{2}
$$

Here, $k_{o}$ and $k_{c}$ are the generalized stiffness of the beam in the fully open crack and the fully closed crack situations, respectively. The parameter $\omega_{b}$ is the bilinear frequency of the free undamped vibration of the cracked beam and can be determined as [22]:

$$
\omega_{b}=2 \frac{\omega_{o} \omega_{c}}{\omega_{o}+\omega_{c}}
$$

Where $\omega_{o}$ and $\omega_{c}$ are the system frequencies in the open crack and the closed crack cases, respectively. Assuming the beam to vibrate predominantly in its fundamental mode, most of the researchers have used an approximate relation to calculate the generalized mass and stiffness of the cantilever beam, which is given by:

$$
\psi(x)=1-\cos \left(\frac{\pi x}{2 L}\right)
$$

In this study to improve the accuracy of the model this admissible function has been replaced by a relation which is obtained analytically by solving the equation of motion of the Euler- Bernoulli beam, that is:

$$
\phi(x)=\cos \lambda x-\cosh \lambda x-\frac{(\cos \lambda L+\cosh \lambda L)}{(\sin \lambda L+\sinh \lambda L)}(\sin \lambda x-\sinh \lambda x)
$$

In this relation, the first mode shape of the cantilever beam is obtained by replacing $\lambda L=1.875$. In Fig. 2 the difference between approximate and analytical mode shapes is demonstrated.

The generalized stiffness of the intact beam in given by [23]:

$$
k_{c}=\frac{1}{C_{c}}=\int_{0}^{L} E I \phi^{\prime \prime 2}(x) d x
$$

Where $C_{c}$ is the flexibility of the intact beam, $E$ is the young's modulus, $I$ is the area moment of inertia of the beam cross section and $L$ is the beam length. From the Eq. (6) it is obvious that the modeling method which is used in this 


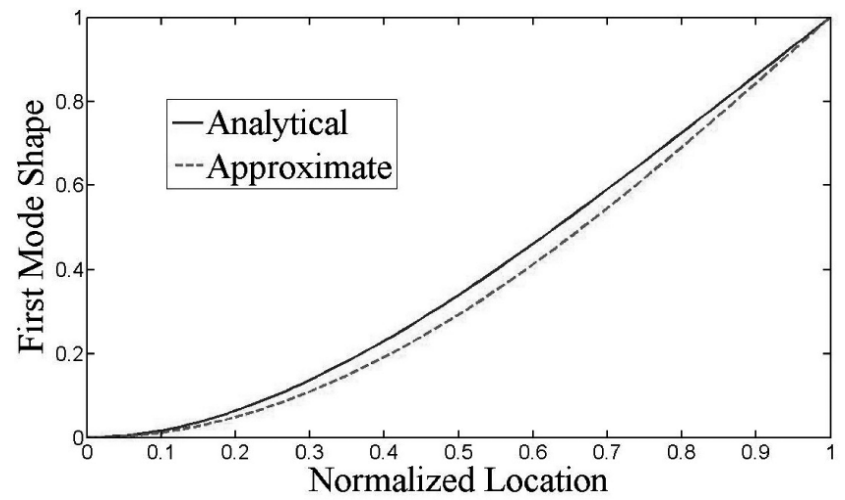

Fig. 2. The analytical (- ${ }_{-}$and the approximate $(---)$first mode shape of the cantilever beam.

study is capable of modeling a beam with any kind of the boundary conditions. The generalized stiffness of a beam with an open crack is given by:

$$
k_{o}=\frac{1}{C_{o}}
$$

Where $C_{o}=C+\Delta C$ and $\Delta C$ is the additional flexibility in the beam due to the presence of the crack. Dimarogonas and Paipetis [24] have determined this value for a cantilever beam with a crack in the distance $l_{c}$ from the free end and the depth of $a$ as:

$$
\Delta C=\frac{72 l_{c}^{2} \pi\left(1-\nu^{2}\right)}{E w b^{4}} \Gamma
$$

Where $w$ and $b$ are the width and the height of the beam cross section, respectively and $\nu$ is the Poisson's ratio. The parameter $\Gamma$ is given by:

$$
\Gamma=19.60 \frac{a^{10}}{b^{8}}-40.69 \frac{a^{9}}{b^{7}}+47.04 \frac{a^{8}}{b^{6}}-32.99 \frac{a^{7}}{b^{5}}+20.30 \frac{a^{6}}{b^{4}}-9.98 \frac{a^{5}}{b^{3}}+4.60 \frac{a^{4}}{b^{2}}-1.05 \frac{a^{3}}{b}+0.63 a^{2}
$$

The generalized mass is given by:

$$
m=\int_{0}^{L} m(x) \phi^{2}(x) d x
$$

Where, $m(x)$ is the mass of the beam per unit length. Finally, the equation of the motion of the SDOF lumped model of a beam with a breathing crack can be expressed as:

$$
m \ddot{u}+c \dot{u}+\left[k_{o}+k_{\Delta}\left(1+\cos \left(\omega_{b} t\right)\right)\right] u=0
$$

Where $u$ is the displacement of the SDOF model which represents the displacement of the free end of the cantilever beam and $c$ is the damping coefficient.

\section{Introducing a new method for solving the equation of motion}

The general form of the free oscillations of most of non-conservative nonlinear systems is given in the form:

$$
\ddot{u}+\omega_{o}^{2} u=\varepsilon f(u, \dot{u}, t)
$$

An approximate solution of such systems can be derived by the method of Multiple Scales which is one of the perturbation methods [25]. In this equation $\omega_{0}$ is the frequency of the equivalent linear system and $\varepsilon$ is a small dimensionless parameter. The Eq. (11) can be written in the form:

$$
\ddot{u}+\left(\frac{k_{o}+k_{\Delta}}{m}\right) u=\zeta\left[-2 \omega_{b} \dot{u}-\frac{2 \omega_{b} k_{\Delta}}{c} \cos \left(\omega_{b} t\right) u\right]
$$


Here $\zeta$ is the damping ratio of the system, which is defined as $\zeta=c /\left(2 m \omega_{b}\right)$. Comparing Eqs (13) and (12) we convert the Eq. (13) to the standard form of Eq. (12) by choosing:

$$
\omega_{0}^{2}=\frac{k_{o}+k_{c}}{2 m}=\frac{\bar{k}}{m}
$$

And

$$
\varepsilon=\zeta
$$

Based on the method of the multiple scales, a solution of Eq. (13) can be represented by the expansion having the form:

$$
u=u_{0}\left(T_{0}, T_{1}\right)+\varepsilon u_{1}\left(T_{0}, T_{1}\right)+\ldots
$$

In this equation the new independent variables can be introduced according to:

$$
T_{n}=\varepsilon^{n} t \quad \text { for } \quad n=0,1, \ldots
$$

So the derivatives of $u$ with respect to the new variables can be expressed as:

$$
\left\{\begin{array}{l}
\dot{u}=D_{0}\left(u_{0}+\varepsilon u_{1}+\ldots\right)+\varepsilon D_{1}\left(u_{0}+\varepsilon u_{1}+\ldots\right)+\ldots \\
\ddot{u}=D_{0}^{2}\left(u_{0}+\varepsilon u_{1}+\ldots\right)+2 \varepsilon D_{0} D_{1}\left(u_{0}+\varepsilon u_{1}+\ldots\right)+\ldots
\end{array}\right.
$$

where $D_{n}$ denotes the partial derivative with respect to $T_{n}$. Substituting Eqs (16) and (18) into Eq. (13) we obtain:

$$
\left[D_{0}^{2}+2 \varepsilon D_{0} D_{1}+\ldots\right]\left[u_{0}+\varepsilon u_{1}+\ldots\right]+\omega_{0}^{2}\left[u_{0}+\varepsilon u_{1}+\ldots\right]=\varepsilon f\left(u_{0}, u_{1}, T_{0}\right) \ldots
$$

By equating coefficients of like powers of $\varepsilon$ to zero, we obtain:

$$
\begin{aligned}
\varepsilon^{0}: & D_{0}^{2} u_{0}+\omega_{0}^{2} u_{0}=0 \\
\varepsilon^{1}: & D_{0}^{2} u_{1}+\omega_{0}^{2} u_{1}=-2 D_{0} D_{1} u_{0}-2 \omega_{b} D_{0} u_{0}-\frac{2 \omega_{b} k_{\Delta}}{c} \cos \left(\omega_{b} T_{0}\right) u_{0}
\end{aligned}
$$

The solution of Eq. (20) can be written in the form:

$$
u_{0}=A\left(T_{1}\right) e^{i \omega_{0} T_{0}}+\bar{A}\left(T_{1}\right) e^{-i \omega_{0} T_{0}}
$$

where $A\left(T_{1}\right)$ is an unknown complex function and $\bar{A}\left(T_{1}\right)$ is complex conjugate of $A\left(T_{1}\right)$. Any particular solution for Eq. (21) would lead to terms that grow without bound as time increases and result in the response to be non-periodic. These terms are called secular terms and can be eliminated if:

$$
-2 D_{0} D_{1} u_{0}-2 \omega_{b} D_{0} u_{0}=0
$$

Therefore, the unknown function $A\left(T_{1}\right)$ is obtained as:

$$
A\left(T_{1}\right)=S e^{-\omega_{b} T_{1}}
$$

where $S$ is determined from initial conditions, and can be assumed in the form:

$$
S=S_{0}+\varepsilon S_{1}+\ldots
$$

Eliminating the secular terms from Eq. (22) and using the method of separation of variables to solve it, one obtains:

$$
u_{1}\left(T_{0}, T_{1}\right)=\left(-\frac{\omega_{b} k_{\Delta}}{c}\right) \cdot\left(e^{-\omega_{b} T_{1}}\right) \cdot\left(\frac{1}{\omega_{0}^{2}-\left(\omega_{b}+\omega_{0}\right)^{2}} \cos \left(\omega_{b}+\omega_{0}\right) T_{0}+\frac{1}{\omega_{0}^{2}-\left(\omega_{b}-\omega_{0}\right)^{2}} \cos \left(\omega_{b}-\omega_{0}\right) T_{0}\right)
$$

Considering only the first correction term, we have:

$$
u\left(T_{0}, T_{1}\right)=e^{-\omega_{b} T_{1}}\left[S \cos \left(\omega_{0} T_{0}\right)+\varepsilon\left(\frac{1}{\omega_{0}^{2}-\left(\omega_{b}+\omega_{0}\right)^{2}} \cos \left(\omega_{b}+\omega_{0}\right) T_{0}+\frac{1}{\omega_{0}^{2}-\left(\omega_{b}-\omega_{0}\right)^{2}} \cos \left(\omega_{b}-\omega_{0}\right) T_{0}\right)\right]
$$

One can write Eq. (27) as a function of time:

$$
u(t)=e^{-\frac{c}{2 m} t}\left[S \cos \left(\omega_{0} t\right)+\varepsilon\left(\frac{1}{\omega_{0}^{2}-\left(\omega_{b}+\omega_{0}\right)^{2}} \cos \left(\omega_{b}+\omega_{0}\right) t_{0}+\frac{1}{\omega_{0}^{2}-\left(\omega_{b}-\omega_{0}\right)^{2}} \cos \left(\omega_{b}-\omega_{0}\right) t\right)\right]
$$

As it is clear from Eq. (28) the response is composed of two parts in which the first part is the response of a system with the mean stiffness of the open crack and the closed crack states and the second part is the first order correction term. 


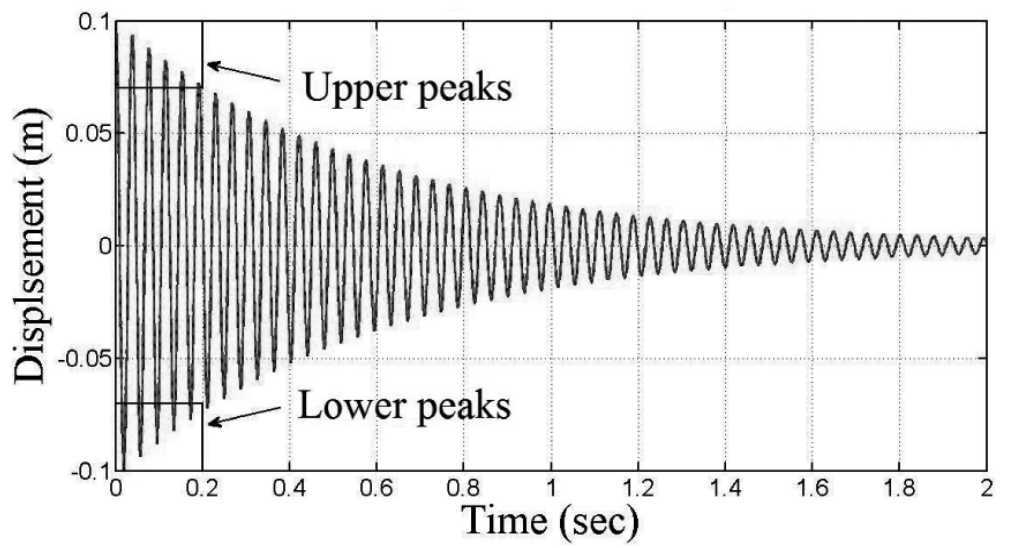

Fig. 3. (a) The first part of the responses obtained through perturbation method $(---)$ and the response obtained with numerical method $(-)$ for $\alpha=0.5$ and $\beta=0.9$.

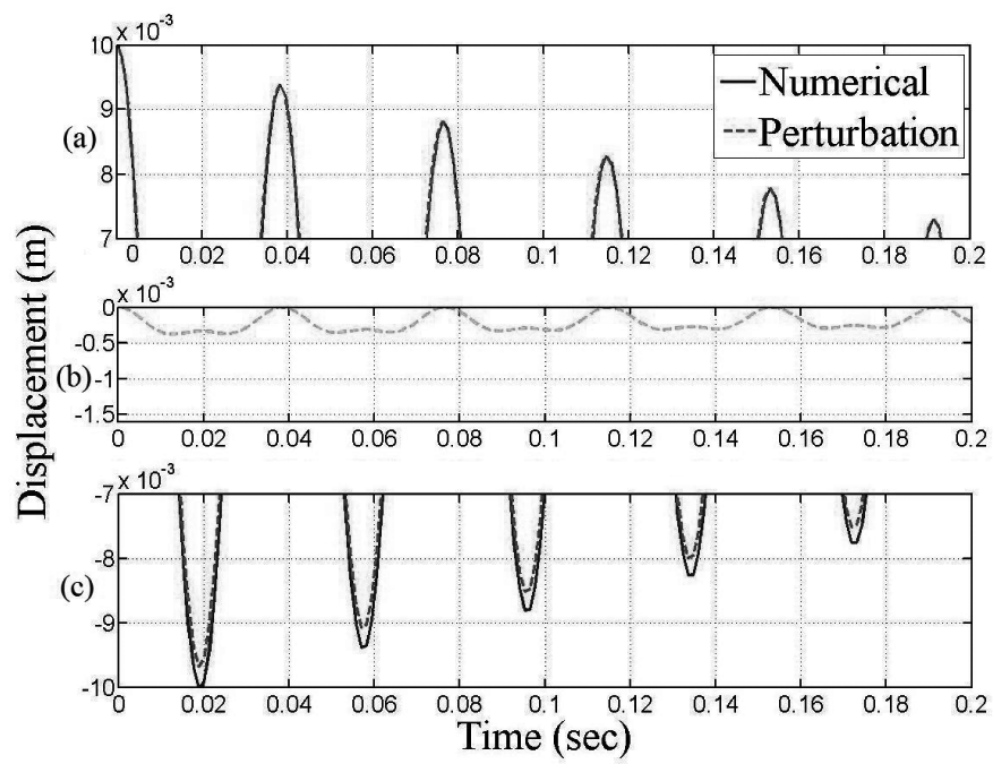

Fig. 4. (a) Response obtained through the perturbation method (- - ) in (a) upper peaks of first part, (b) second part and (c) lower peaks of first part and numerical response (- $(-)$ for $\alpha=0.5$ and $\beta=0.9$.

\section{Theoretical results}

Consider a rectangular cantilever beam containing a transverse breathing crack, as shown in Fig. 1. The dimensions are chosen as: $L=450 \mathrm{~mm}, w=3.9 \mathrm{~mm}$ and $b=6.4 \mathrm{~mm}$. Young's modulus is $E=200 \mathrm{GPa}$ and density is $\rho=$ $7860 \mathrm{~kg} / \mathrm{m}^{3}$. The dimensionless crack parameters are $\alpha=\frac{a}{b}$ and $\beta=\frac{l_{c}}{L}$, where $l_{c}$ is the crack position measured form the free end of the beam and $a$ is the crack depth. The damping ratio is taken $\zeta=0.01$.

In the previous section, it has been shown that the calculated response is composed of two parts. In Fig. 3 the first part of perturbation response together with the numerically obtained response using the Rung-Kutta method are shown.

Figure 4 zooms over the upper peaks and the lower peaks of the time history in Fig. 3. It also shows the second part of perturbation response which is a correction term and will be added to the first part.

From Fig. 4-a and c it can be seen that the main part of the perturbation response and the numerical response are coincided in the upper peaks, while in the lower peaks they are different. This difference stems from decrease in 


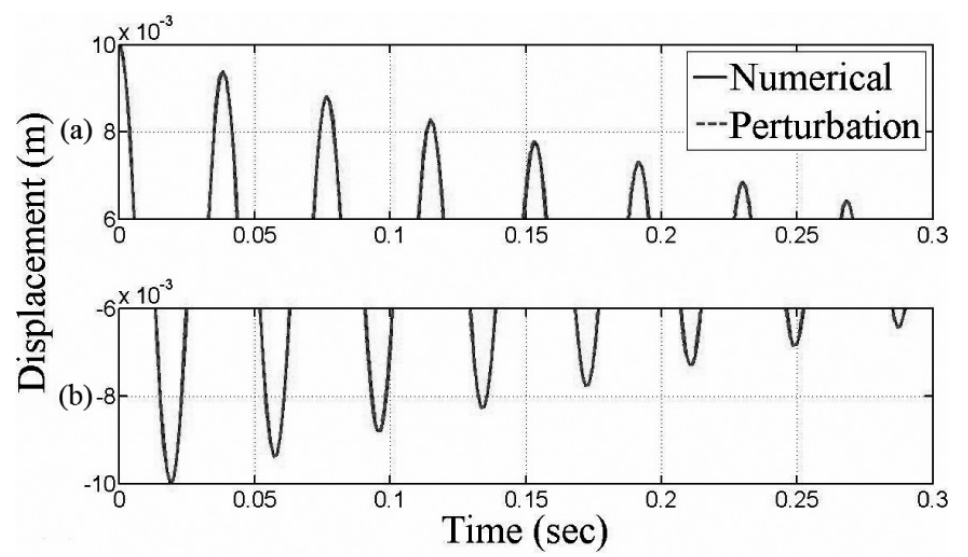

Fig. 5. (a) Responses obtained through the perturbation method (- - -) and numerical method (-----) in (a) upper half cycles, (b) lower half cycles.

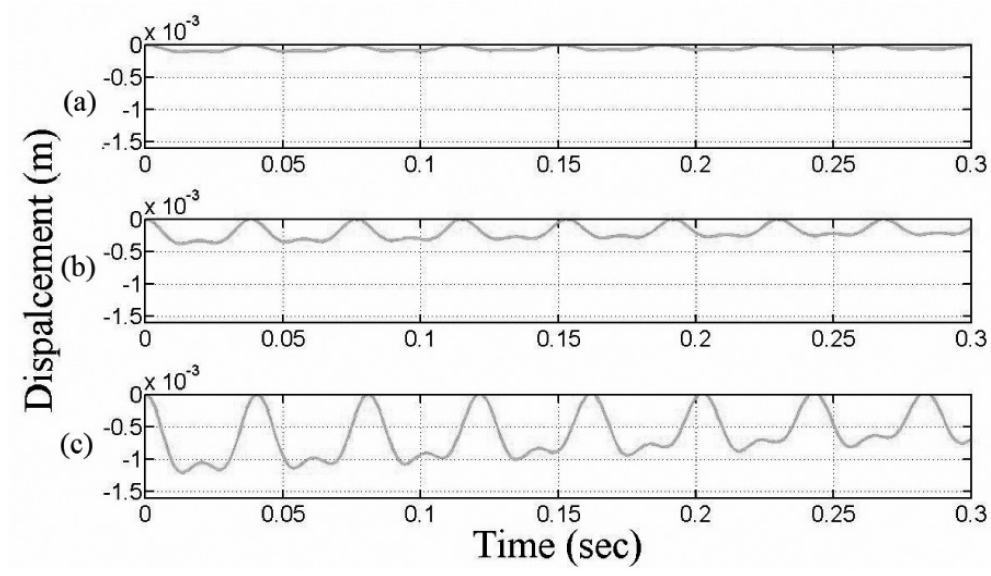

Fig. 6. Correction term for $\beta=0.9$ and (a) $\alpha=0.3$, (b) $\alpha=0.5$, (c) $\alpha=0.7$.

the system's stiffness due to the crack opening in the lower half cycles. Figure 4-b illustrates the correction term of perturbation response. It is obvious that this part of the response contains the influence of the breathing crack which mainly affects the system response in lower half cycles. After addition of correction term to the main part, the total perturbation response coincides with the numerical response on both upper peaks and lower peaks (Fig. 5).

The increase of the crack depth results in increasing the effect of breathing crack on the response which is reflected by correction term. This phenomenon is revealed in Fig. 6.

The correlation between the variation range of the correction term and the normalized crack position is shown in Fig. 7. It can be seen that for a certain value of the crack depth, the influence of the correction term increases by approaching the crack to the clamped end.

The ratio of the correction term to the main part of the response as a function of normalized crack parameters is depicted in a three dimensional diagram in Fig. 8.

Figure 8 can be used for predicting the relative crack depth and its location.

\section{Experimental results}

To validate the theoretical results, some experimental tests were carried out on a cracked steel beam with structural damage. In most studies carried on damaged structures, the crack in the structure has been created using a thin saw 
Table 1

A compression between measured and predicted 1st natural frequency

of the cracked cantilever beam with $\alpha=0.38$ and $\beta=0.8$

\begin{tabular}{lcc}
\hline & Intact Beam $(\mathrm{Hz})$ & Cracked Beam $(\mathrm{Hz})$ \\
\hline Experimental & 23.500 & 23.375 \\
Perturbation & 23.446 & 23.217 \\
Analytical (open crack) & 23.446 & 22.992 \\
\hline
\end{tabular}

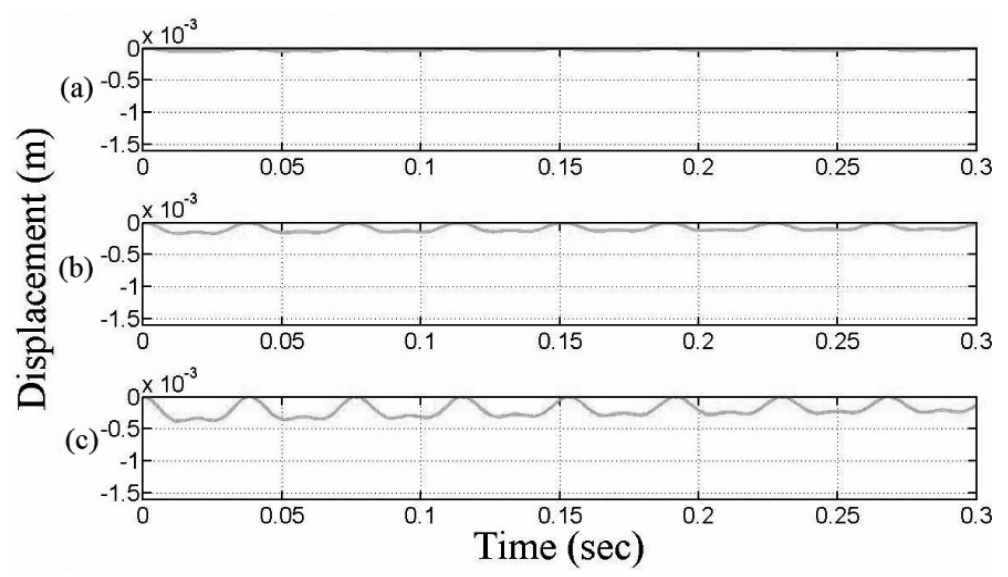

Fig. 7. Correction term for $\alpha=0.5$ and (a) $\beta=0.3$ (b) $\beta=0.6$ (c) $\beta=0.9$.

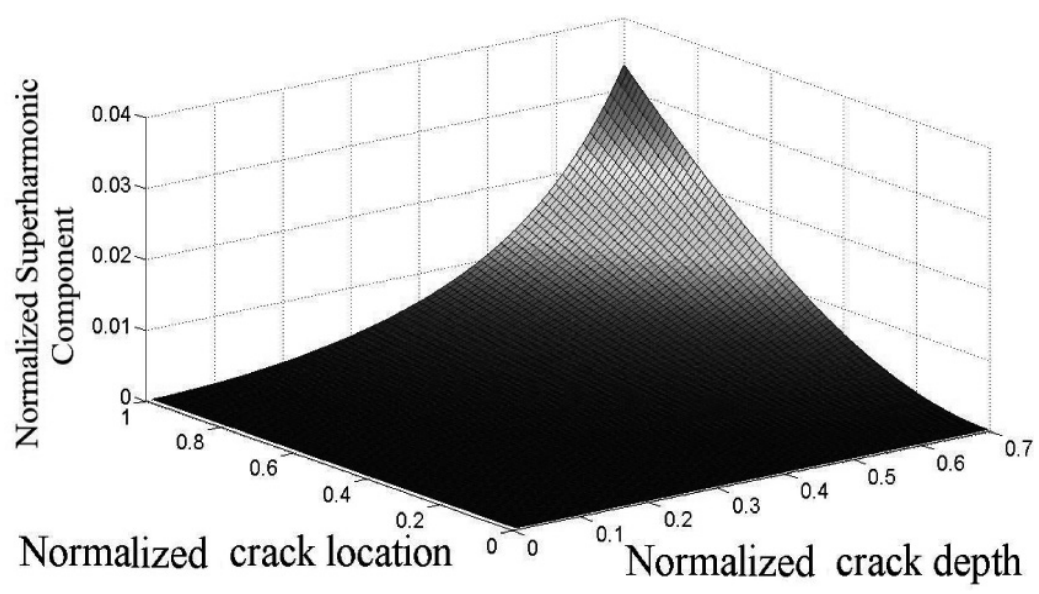

Fig. 8. The ratio of the correction term to the main part of response.

cut. But attempts to simulate a breathing crack by saw cuts failed to provide the adequate breathing performance of a real fatigue crack [26]. In this study a Zwick/Roell servo-hydraulic testing machine (model Amsler HA250), was used for creating and growing a fatigue crack in the specimen. Geometrical dimensions and mechanical properties of the beam are the same as the one which studied in the previous section while the crack parameters are $\alpha=0.38$ and $\beta=0.8$.

The beam is considered to be excited by displacing the free end and then releasing it. The dynamic response of the beam was captured using an accelerometer (4507 B \& K) attached to the free end. The response measurements were acquired using signal analyzer (3109 B \& K). A schematic of the experimental set-up is depicted in Fig. 9

In Table 1, the measured natural frequencies of the intact and the cracked beams are compared to the perturbation values and the analytically obtained ones with open crack assumption. As it has been previously proven in the literature, it is obvious that the reduction in system natural frequency due to a certain breathing crack is considerably 


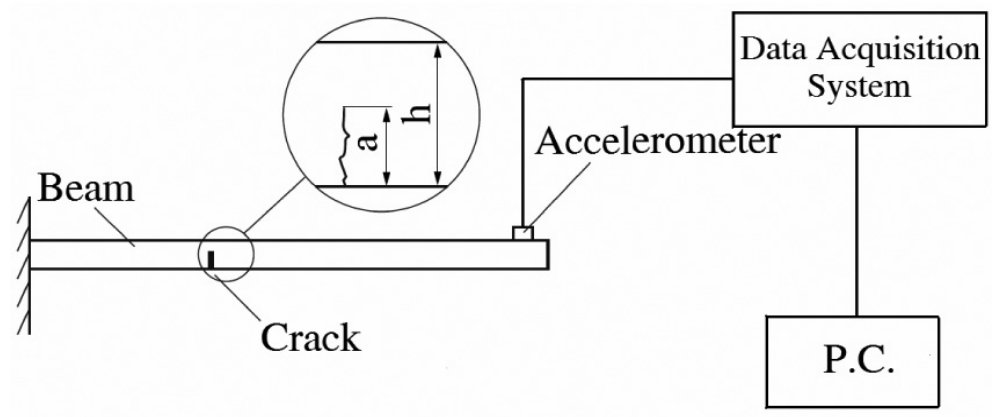

Fig. 9. Set-up of the experimental apparatus.

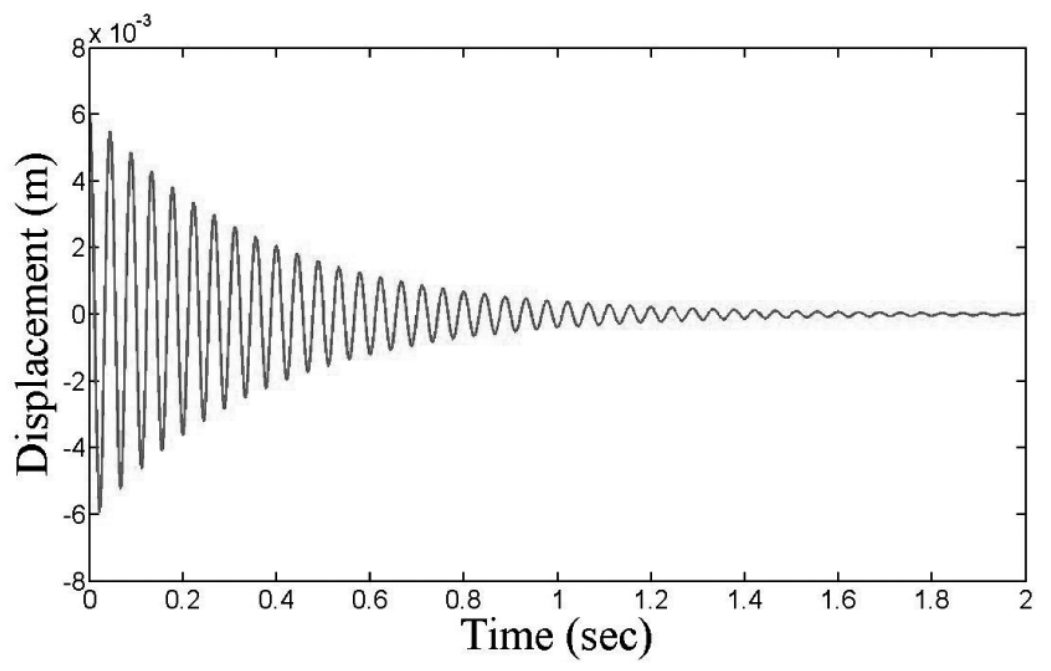

Fig. 10. Experimental time history of the cracked cantilever beam with $\alpha=0.38$ and $\beta=0.8$.

less than that predicted by linear open crack assumption. This fact, if ignored in vibration based crack detection methods, can lead to catastrophic results. To extract the experimental cracked beam natural frequency, the tests are repeated for three times at the identical test conditions, and at each time, by acquiring the time history of the cracked beam, the natural frequency is obtained by locating the fundamental peak on the calculated spectrum. Using this procedure, the natural frequency of the beam is obtained as $23.350(\mathrm{~Hz}), 23.410(\mathrm{~Hz})$ and $23.365(\mathrm{~Hz})$, with the average value of $23.375(\mathrm{~Hz})$.

In Fig. 10, the experimental time history of the cracked beam is shown. The fatigue crack not only affects the structural stiffness, but also it influences the structural damping [9]. The damping ratio for the mentioned cracked beam is obtained as $\zeta=0.02$.

In Fig. 11, the spectrum of the measured response of the cracked beam is shown. Comparing to Fig. 12 which is the frequency spectrum of the intact beam; reveals that the appearance of the first superharmonic component is due to the presence of the breathing crack. This phenomenon can be described by considering the results of the perturbation analysis of this study. Due to Eq. (16) the perturbation response is composed of two terms, the main part and the correction term (of $\varepsilon$ order). Since the correction term has a superharmonic component due to Eq. (26), the presence of the first superharmonic in the spectrum stems from this part of the response. In this study $\varepsilon=\zeta$, so when the crack depth increases, $\omega_{o}$ and $\omega_{c}$ get away from each other which results in reduction of $\omega_{b}$ due to Eq. (3). Consequently, the dimensionless parameter of $\zeta=c /\left(2 m \omega_{b}\right)$ increases and the effect of the first superharmonic in the response increases. Due to the weak nonlinearity of the system, the magnitude of other harmonics is quite small.

In Fig. 13 frequency spectrum of the theoretical response of the cracked beam is shown. By comparing the Figs 11 and 13 , it is concluded that there is a good agreement between the theoretical and experimental results. 


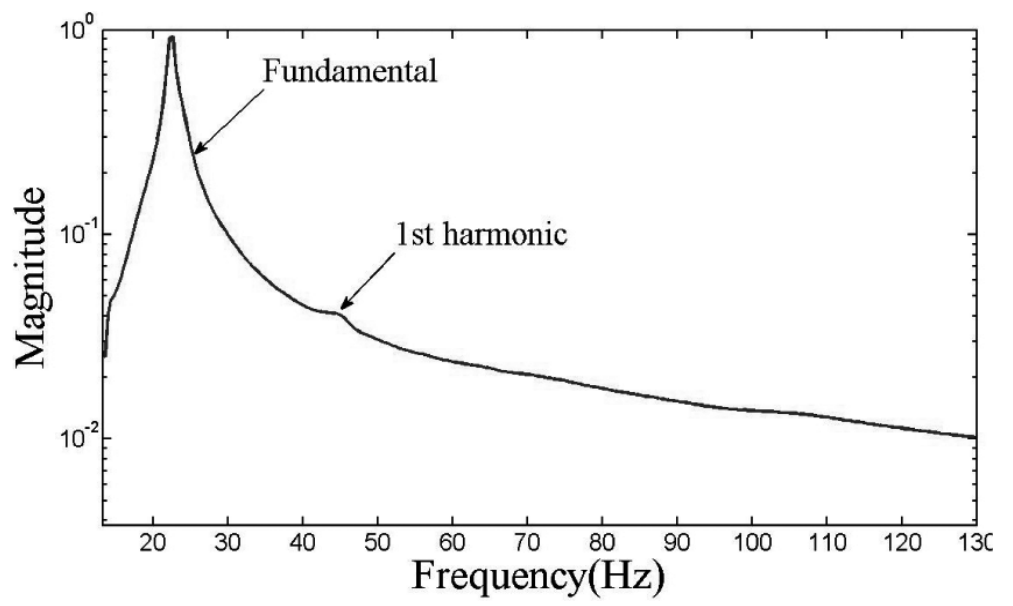

Fig. 11. Spectrum of the measured response of the cracked cantilever beam with $\alpha=0.38$ and $\beta=0.8$.

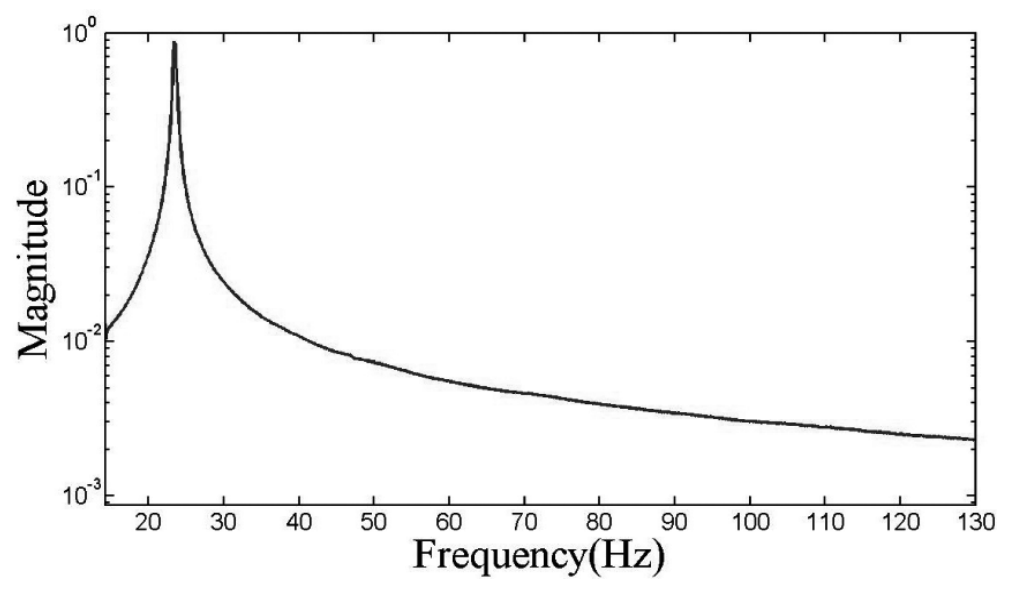

Fig. 12. Spectrum of the measured response of the intact beam.

\section{Results and discussion}

The nonlinear vibration behavior of a cantilever beam with a breathing crack was investigated both theoretically and experimentally. For the sake of simplicity only free vibrations of the beam were considered. The primary aim was to highlight the nonlinear characteristics of the response of a cracked beam by using a perturbation analysis instead of the traditional numerical methods. For this purpose, first the lumped equation of motion of the cracked beam converted to the standard form which can be solved by the perturbation method. Then using the multiple scales method the response was obtained. It was found that the correction term of the calculated response exhibits a temporal behavior oscillating between zero and a maximum value which decays by time due to the damping. Thus, the effect of the crack breathing behavior on system response not only in frequency domain but also in time domain is captured and revealed.

Moreover, this part of the response relates the crack parameters to the first and the most important superharmonic of response. As a result, this method not only can be used for investigating nonlinear vibration behavior of cracked beam but also due to its considerably low computational cost, it has the potential of being used in developing the crack detection indexes in the inverse problem.

It was shown that the ratio of the superharmonic part to the main response increases with crack parameters following a three dimensional surface. Also, the presence of the superharmonic as an indicator of nonlinearity was confirmed by the experimental tests. The vibration tests have been performed on the beam with a real fatigue crack. 


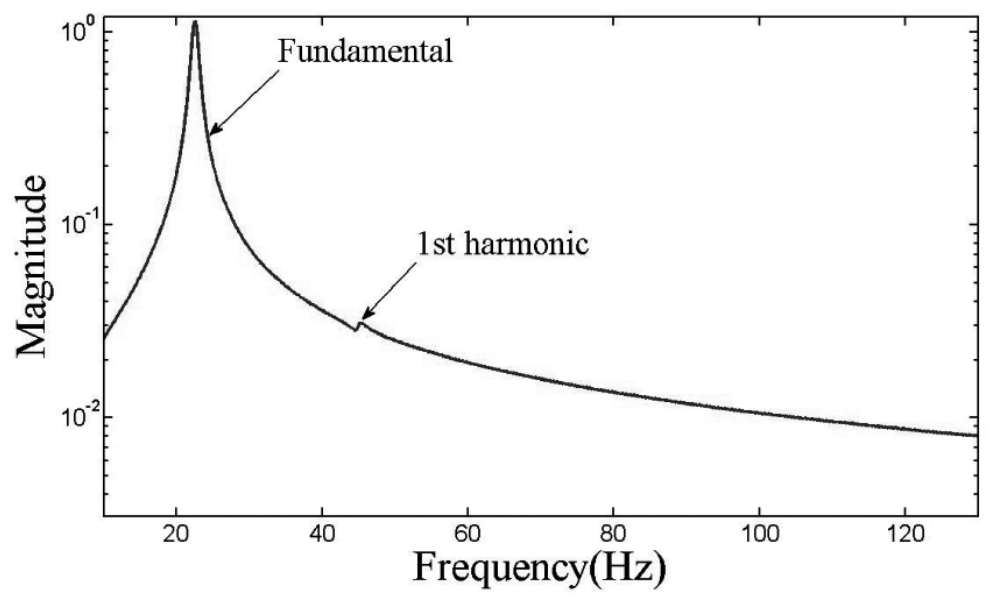

Fig. 13. Spectrum of the cracked beam obtained theoretically.

Therefore, we employed the smooth breathing assumption i.e. gradual stiffness variation between the two extremes, and the good agreement between theoretical and experimental results stems from this realistic assumption in the employed model.

The presented results provide a foundation of using perturbation method as an efficient tool for the description of the nonlinearities caused by the breathing crack and consequently, it can be used to improve the accuracy of the vibration based crack detection techniques.

\section{References}

[1] S.W. Doebling, C.R. Farrar, M.B. Prime and D.W. Shevitz, Damage identification and health monitoring of structural and mechanical systems for changes in their vibration characteristics: a literature review, Report no: LA-13070-MS, Los Alamos Laboratory, 1996.

[2] R. Ruotolo, C. Surace, P. Crespo and D.M. Storer, Harmonic analysis of the vibrations of a cantilevered beam with a closing crack, Computers and Structures 61 (1996), 1057-1074.

[3] P. Gudmunson, The dynamic behaviour of slender structures with cross-sectional cracks, Journal of mechanics and physics of solids 31 (1983), 329-345.

[4] J.C. Newman and W. Elber, A comparison of measurement methods and numerical procedures for the experimental characterization of fatigue crack closure, Mechanics of fatigue crack closure, ASTM, 1989, pp. 171-185.

[5] S.M. Cheng, X.J. Wu, W. Wallace and A.S.J. Swamidas, Vibrational response of a beam with a breathing crack, Journal of Sound and Vibration 225 (1999), 201-208.

[6] P.G. Kirmsher, The effect of discontinuities on the natural frequency of beams, Proceedings of the American Society of testing and Materials 44(1944), 897-904.

[7] A. Ibrahim, F. Ismail and H.R. Martin, Modeling of the dynamics of a continuous beam including nonlinear fatigue crack, International Journal of Analytic and Experimental Modal Analysis 2 (1987), 76-82.

[8] R.I. Actis and A.D. Dimarogonas, Non-linear effects due to closing cracks in vibrating beams, 12th ASME Conference on Mechanical Engineering, vibration and Noise, Montreal, Canada, 17-20 September 1989.

[9] A.P. Bovsunovskii, Vibrations of a non-linear mechanical system simulating a cracked body, Strength of Materials 33(4) (2001), 370-379.

[10] T.G. Chondros, A.D. Dimarogonas and J. Yao, Vibration of a beam with a breathing crack, Journal of Sound and Vibration 239(1) (2001), 57-67.

[11] E. Luzzato, Approximate computation of non-linear effects in a vibrating cracked beam, Journal of Sound and Vibration 265(4) (2003), 745-763.

[12] M.I. Friswell and J.E.T. Penny, A simple nonlinear model of a cracked beam, Proceedings of 10th International Modal Analysis Conference, 1992, pp. 516-521.

[13] M.H.H. Shen and Y.C. Chu, Vibrations of beams with a fatigue crack, Journal of Computers and Structures 45 (1992), 79-93.

[14] M.H.H. Shen and Y.C. Chu, Analysis of forced bilinear oscillators and the application to cracked beam dynamics, AIAA Journal 30 (1992), 2512-2519.

[15] W.M. Ostachowicz and M. Krawczuk, Forced vibrations of a cantilever Timoshenko beam with a closing crack, Proceedings of ISMA 19, Leuven, Belgium 3 (1994), 1067-1078.

[16] P.N. Saavedra and L.A. Cuitino, Crack detection and vibration behavior of cracked beams, Journal of Computers and Structures 79 (2001), 1451-1469. 
[17] J.K. Sinha and M.I. Friswell, Simulation of the dynamic response of a cracked beam, Journal of Computers and Structures 80 (2002), $1473-1486$.

[18] U. Andreaus, P. Casini and F. Vestroni, Non-linear dynamics of a cracked cantilever beam under harmonic excitation, International Journal of Non-Linear Mechanics 42 (2007), 566-575.

[19] R. Clark, W.D. Dover and L.J. Bond, The effect of crack closure on the reliability of NDT predictions of crack size, NDE\&E International 20 (1987), 269-275.

[20] E. Douka and J.L. Hadjileontiadis, Time-frequency analysis of the free vibration response of a beam with a breathing crack, $N D T \& E$ International 38 (2005), 3-10.

[21] Z.K. Peng, Z.Q. Lang and S.A. Billings, Crack detection using nonlinear output frequency functions, Journal of Sound and Vibration 301 (2007), 777-788.

[22] Y.S. Choi and S.T. Noah, Forced periodic vibration of unsymmetric piecewise-linear systems, Journal of Sound and Vibration 121 (1988), $117-126$.

[23] W.R. Clough and J. Penzien, Dynamics of Structures, McGraw-Hill, New York, 1975.

[24] A.D. Dimarogonas and S.A. Paipetis, Analytical Methods in Rotor Dynamics, Applied Science Publishers, 1983.

[25] A.H. Nayfeh and D.T. Mook, Nonlinear oscillations, John Wiley and Sons, 1979.

[26] A.D. Dimarogonas, Vibration of cracked structures: a state of the art review, Engineering Fracture Mechanics 55 (1996), 831-857. 

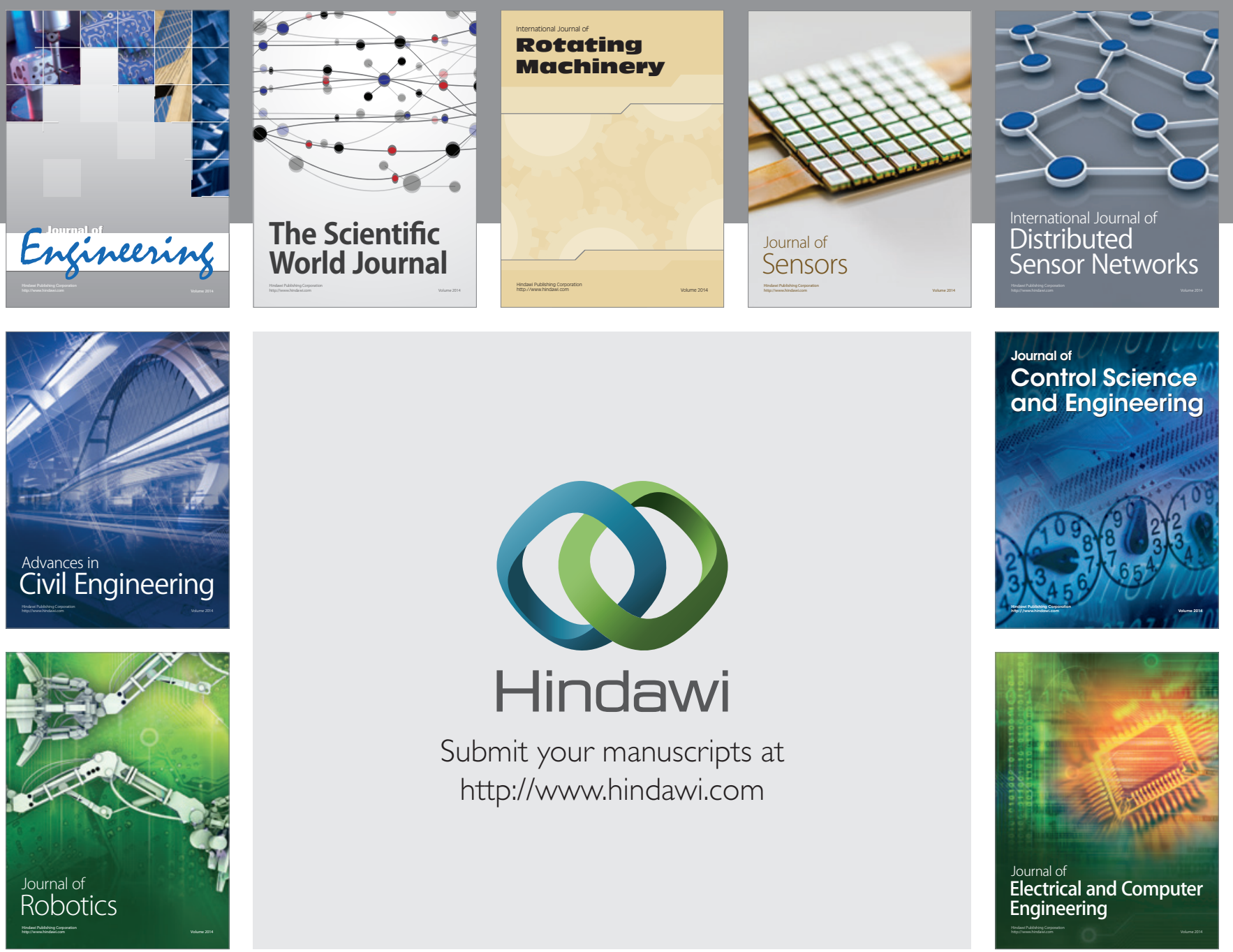

Submit your manuscripts at

http://www.hindawi.com
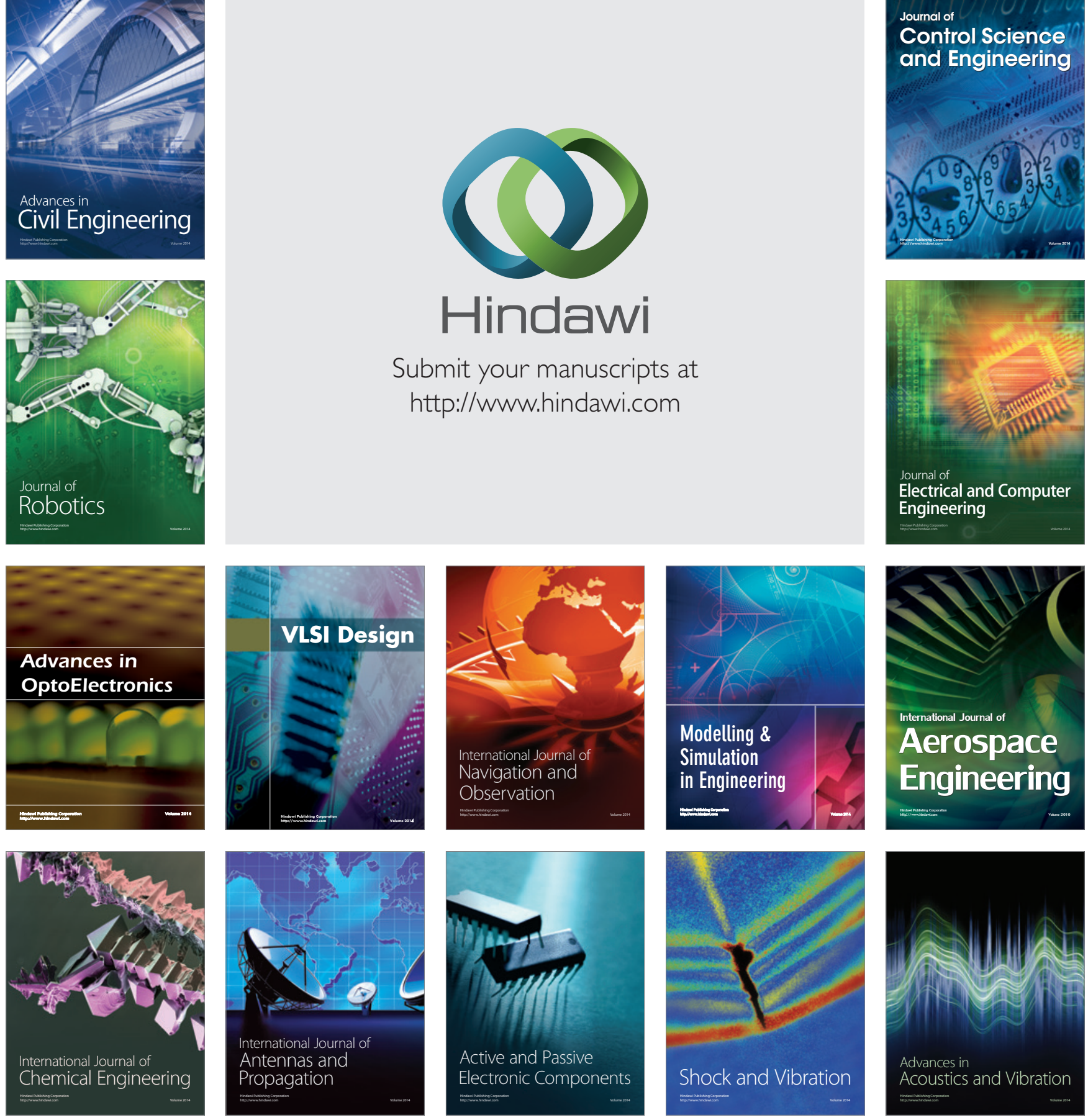